\title{
A NOTE ON POLYNOMIAL OPERATOR APPROXIMATION
}

\author{
R. R. SMITH AND J. D. WARD
}

\begin{abstract}
An example is given of an operator $T$ contained in a block-diagonal algebra of operators $Q$, an ideal $J \subset Q$ and an infinite set of polynomials ${ }^{\circ}$ for which there is a $K \in J$ satisfying $\|p(T+K)\|_{Q \mathcal{Q}}=\|p(T+K)\|_{Q / J}$ for any finite subset of ( $P$ but for which there is no $K \in J$ satisfying $\|p(T+K)\|_{Q^{P}}=\|p(T+K)\|_{Q^{\prime} / J}$ for all $p \in$ ? $P$. This sheds some light on a well-known question of C. Olsen.
\end{abstract}

In her work on compact perturbations of operators Olsen [3] proposed (and solved in various special cases) the following problem: given $T \in B(H)$ and a polynomial $p(x)$ does there exist a compact operator $K$ so that $\|p(T+K)\|=\|p(T)\|_{e}$, where $\|\cdot\|_{e}$ denotes the norm in the Calkin algebra? This question was taken up for a $C^{*}$-algebra with a closed ideal by Akemann and Pedersen [1] where it was shown that perturbations can be chosen to given equality for the polynomial $x^{n}$. A counterexample was given for the polynomial $x^{2}-x$ in the algebra $C[0,1]$ of continuous functions on the unit interval. The purpose of this note is to give an example of a $C^{*}$-algebra $A$, an ideal $J$, an infinite collection of polynomials and an operator $T \in A$ so that a perturbation may be found for any finite number of the polynomials, but no perturbation exists for all of the polynomials simultaneously.

Let $S_{n}$ be the upper triangular $n \times n$ matrix with l's on and above the main diagonal. It is easy to see that powers of $S_{n}$ are also upper triangular and are constant on the diagonals. Let $\alpha_{l}^{(r)}$ denote the $(1, l)$ entry of $\left(S_{n}\right)^{r}$. The following lemma will be useful in evaluating $\alpha_{l}^{(r)}$.

LEMMA 1. $\sum_{i=1}^{m} i^{k}$ is a polynomial $\beta_{k}(m)$ in $m$ whose leading term is $(k+1)^{-1} m^{k+1}$.

Proof. Since $\sum_{i=1}^{m} i=m(m+1) / 2$, the result is true for $k=1$. Assume the result for $k \leqslant r-1$ and consider the identity

$$
m^{r+1}-1=\sum_{i=1}^{m-1}\left[(1+i)^{r+1}-i^{r+1}\right] \text {. }
$$

Expansion of $(1+i)^{r+1}$ combined with the induction hypothesis results in

$$
m^{r+1}-1=(r+1) \sum_{i=1}^{m-1} i^{r}+q(m)
$$

Received by the editors November 3, 1981 and, in revised form, August 5, 1982.

1980 Mathematics Subject Classification. Primary 47A30; Secondary 47A65.

Key words and phrases. Block diagonal operator. 
where $q(m)$ is a polynomial of degree $r$. Add $(r+1) m^{r}$ to both sides to obtain

$$
\sum_{i=1}^{m} i^{r}=(r+1)^{-1} m^{r+1}-(r+1)^{-1}\left[1+q(m)-(r+1) m^{r}\right],
$$

and the induction step is proved.

LEMMA 2. For $r \geqslant 2, \alpha_{l}^{(r)}$ is obtained by evaluating at $x=$ l a polynomial $p_{r}(x)$ whose leading term is $x^{r-1} /(r-1)$ !.

Proof. To begin the induction argument observe that $\alpha_{l}^{(2)}=l, 1 \leqslant l \leqslant n$, and so $p_{2}(x)=x$. Now assume the truth of the lemma for all integers less than or equal to $r$ and write

$$
p_{r}(x)=\sum_{i=0}^{r-1} \lambda_{i, r} x^{i}
$$

where $\lambda_{r-1, r}=1 /(r-1)$ !. It follows from the identity $\left(S_{n}\right)^{r+1}=\left(S_{n}\right)^{r} S_{n}$ that

$$
\begin{aligned}
\boldsymbol{\alpha}_{q}^{(r+1)} & =\sum_{k=1}^{q} \boldsymbol{\alpha}_{k}^{(r)}=\sum_{k=1}^{q} \sum_{i=0}^{r-1} \lambda_{i, r} k^{i} \\
& =\sum_{i=0}^{r-1}\left(\lambda_{i, r} \sum_{k=1}^{q} k^{i}\right)=\sum_{i=0}^{r-1} \lambda_{i, r} \beta_{i}(q)
\end{aligned}
$$

by Lemma 1. Then $\alpha_{q}^{(r+1)}$ is given by evaluation of the polynomial $p_{r+1}(x)=$ $\sum_{i=0}^{r-1} \lambda_{i, r} \beta_{i}(x)$. The degree of $p_{r+1}(x)$ is $r$ and the leading term is $\lambda_{r-1, r} x^{r} / r$, which is $x^{r} / r$ !.

LEMMA 3. For each fixed positive integer $r \geqslant 2$,

$$
\limsup _{n \rightarrow \infty} \frac{\left\|\left(S_{n}\right)^{r}\right\|}{n^{r}} \leqslant \frac{(2 r-1)^{-1 / 2}}{(r-1) !} .
$$

Proof. Let $\left\{\mathbf{x}_{i}\right\}_{i=1}^{n}$ be the $n$ rows of $\left(S_{n}\right)^{r}$ and let $\mathbf{y}$ be a unit vector in $\mathbf{C}^{n}$ at which $\left(S_{n}\right)^{r}$ attains its norm. The entries of $\mathbf{y}$ may be taken as nonnegative since the entries of $\left(S_{n}\right)^{r}$ are nonnegative. The $i$ th entry of $\left(S_{n}\right)^{r} \mathbf{y}$ is $\left\langle\mathbf{x}_{i}, \mathbf{y}\right\rangle$ and so

$$
\begin{aligned}
\left\|\left(S_{n}\right)^{r}\right\| & =\left\|\left(S_{n}\right)^{r} \mathbf{y}\right\|=\left(\sum_{i=1}^{n}\left\langle\mathbf{x}_{i}, \mathbf{y}\right\rangle^{2}\right)^{1 / 2} \\
& \leqslant\left(\sum_{i=1}^{n}\left\|\mathbf{x}_{i}\right\|^{2}\|\mathbf{y}\|^{2}\right)^{1 / 2}=\left(\sum_{i=1}^{n}\left\|\mathbf{x}_{i}\right\|^{2}\right)^{1 / 2} \leqslant n^{1 / 2}\left\|\mathbf{x}_{1}\right\|
\end{aligned}
$$

since $\left\|\mathbf{x}_{i}\right\| \leqslant\left\|\mathbf{x}_{1}\right\|$ for $1 \leqslant i \leqslant n$. Consequently

$$
\left\|\left(S_{n}\right)^{r}\right\|^{2} \leqslant n \sum_{i=1}^{n}\left(\alpha_{i}^{(r)}\right)^{2}=n \sum_{i=1}^{n}\left(p_{r}(i)\right)^{2}
$$

and this in turn is a polynomial in $n$ whose leading term is $n^{2 r} /(r-1) !^{2}(2 r-1)$ from Lemmas 1 and 2 . It follows that (1) holds. 
LemMA 4. Let $T$ be an operator whose numerical range $W(T)$ contains 0 and let $D$ be an operator satisfying $0 \leqslant D \leqslant I$. Then, for all $\lambda \in \mathbf{C}$.

$$
\|\lambda+D T D\| \leqslant\|\lambda+T\| .
$$

Proof. Let $\theta$ be a state on $B(H)$ satisfying $\theta(T)=0$, and define a completely positive unital map $\psi: B(H) \rightarrow B(H)$ by $\psi(X)=\theta(X)\left(I-D^{2}\right)+D X D$. Then $\|\psi\|=1$ and $\psi(\lambda+T)=\lambda+D T D$. It follows that, for all $\lambda \in \mathbf{C},\|\lambda+D T D\| \leqslant$ $\|\lambda+T\|$.

It is now possible to describe the example mentioned above. Let $M_{n}$ denote the $n \times n$ matrix algebra and let $A$ be the $C^{*}$-algebra $\bigoplus_{n=1}^{\infty} M_{n}$, which can be viewed as block-diagonal operators on a separable Hilbert space. Elements of $A$ may be written $T=\bigoplus_{n=1}^{\infty} T_{n}$ where it is understood that $T_{n} \in M_{n}$. Let $J$ denote the ideal of compact block-diagonal operators. For an operator $T \in A$ its norm in the quotient space will be denoted by $\|T\|_{e}$, and its image under the quotient map by $\dot{T}$.

Recall the definition of the matrix $S_{n}$, and define $T=\bigoplus_{n=1}^{\infty} S_{n} / n \in A$. It follows from Lemma 3 that $\left\|T^{r}\right\|_{e} \leqslant(2 r-1)^{-1 / 2} /(r-1)$ ! and so

$$
\lim _{r \rightarrow \infty}\left(\left\|T^{r}\right\|_{e}\right)^{1 / r} \leqslant \lim _{r \rightarrow \infty} \frac{(2 r-1)^{-1 / 2 r}}{((r-1) !)^{1 / r}}=0 .
$$

Therefore $\dot{T}$ is quasinilpotent.

Let $P_{n} \in M_{n}$ be the projection all of whose entries are $1 / n$, and write $P=\bigoplus_{n=1}^{\infty} P_{n}$ $\in A$. Then $\dot{P}$ and $\operatorname{Re}(\dot{T})$ are equal and so the numerical range $W(\dot{T})$ of $\dot{T}$ lies in the right half-plane.

THEOREM 1. Given any finite number, $q_{1}(x), \ldots, q_{l}(x)$, of polynomials from either of the collections $\left\{x^{n}: n \geqslant 1\right\}$ or $\{x+\lambda: \lambda \in \mathbf{C}\}$ there exists an element $K \in J$ so that

$$
\left\|q_{i}(T)\right\|_{e}=\left\|q_{i}(T+K)\right\|, \quad 1 \leqslant i \leqslant l .
$$

However no such $K$ exists for which equality holds simultaneously for all of these polynomials.

Proof. First choose $K_{1} \in J$ so that $\left\|\lambda+\left(T+K_{1}\right)\right\|=\|\lambda+T\|_{e}$ for all $\lambda \in \mathbf{C}$ [5, Theorem 1.2, Corollary 1.3]. For a fixed integer $n$ there exists $C \in J$ so that $0 \leqslant C \leqslant I$ and $\left\|\left[\left(T+K_{1}\right)(I-C)\right]^{r}\right\|=\left\|T^{r}\right\|_{e}$ for $1 \leqslant r \leqslant n$ [1, Theorem 3.7]. The element $C$ in the proof of this theorem is constructed from any quasicentral approximate identity of $J$. In this case a diagonal approximate identity may be chosen which is constant on the blocks and so $(I-C)$ commutes with $\left(T+K_{1}\right)$. Write $D=(I-C)^{1 / 2}, 0 \leqslant D \leqslant I$, and observe that $D\left(T+K_{1}\right) D$ is a compact perturbation of $T$ by some $K \in J$. Then

$$
\left\|(T+K)^{r}\right\|=\left\|\left[D\left(T+K_{1}\right) D\right]^{r}\right\|=\left\|\left[\left(T+K_{1}\right)(1-C)\right]^{r}\right\|=\left\|T^{r}\right\|_{e}
$$

for $1 \leqslant r \leqslant n$.

The operator $T$ is essentially quasinilpotent, by construction, and so $0 \in$ $W\left(T+K_{1}\right)$. By Lemma 4

$$
\|\lambda+(T+K)\|=\left\|\lambda+D\left(T+K_{1}\right) D\right\| \leqslant\left\|\lambda+\left(T+K_{1}\right)\right\|=\|\lambda+T\|_{e}
$$


and inequality in the opposite direction is immediate. Thus, for any finite collection of polynomials, the existence of an appropriate $K \in J$ is established.

Suppose, on the other hand, that there exists $K \in J$ so that

$$
\left\|T^{r}\right\|_{e}=\left\|(T+K)^{r}\right\|, \quad r \geqslant 1,
$$

and

$$
\|T+\lambda\|_{e}=\|T+K+\lambda\|, \quad \lambda \in \mathbf{C} .
$$

This would imply that $T+K$ is quasinilpotent and that $W(T+K)=W_{e}(T)$ [6, Theorem 4] and hence lies in the right half-plane. If $T+K$ is written $\bigoplus_{n=1}^{\infty} R_{n}$, then each $R_{n}$ is nilpotent and each $W\left(R_{n}\right)$ lies in the right half-plane. Thus, for each $n, 0$ is on the boundary of the numerical range of the nilpotent matrix $R_{n}$. Thus $R_{n}$ is identically zero [2, Theorem 2], which is impossible since $T+K$ is a compact perturbation of the noncompact operator $T$. This contradiction completes the proof.

Acknowledgement. The authors wish to thank D. D. Rogers for a helpful suggestion.

\section{REFERENCES}

1. C. A. Akemann and (J. K. Pedersen, Ideal perturbations of elements in $C^{*}$-algehras, Math. Scand. 41 (1977), 117-139.

2. W. F. Donoghue, On the numerical range of a bounded operator, Michigan Math. J. 4 (1957), 261-263.

3. C. L. Olsen, Norms of compact perturbations of operators, Pacific J. Math. 68 (1977), 209-228.

4. C. L. Olsen and J. K. Plastiras, Quasialgebraic operators, compact perturbations and the essential norm, Michigan Math. J. 21 (1974), 385-397.

5. R. R. Smith and J. D. Ward, Locally isometric liftings from quotient $C^{*}$-algebras, Duke Math. J. 47 (1980), 621-631.

6. J. G. Stampfli and J. P. Williams, Growth conditions and the numerical range in a Banach algebra, Tôhoku Math. J. 20 (1968), 417-424.

Department of Mathematics, Texas A \& M University, College Station, Texas 77843 